\title{
The effect of two root canal sealers on the retentive strength of glass fibre endodontic posts
}

\author{
S. T. DAVIS \& B. C. O'C ONNELL School of Dental Science, Trinity College, Dublin, Ireland
}

SUMMARY This study was undertaken to examine the effect of eugenol-containing and non-eugenol-containing root canal sealers on the retention strength of glass fibre endodontic posts (ParaPost Fibre White) luted with a resin cement (ParaPost cement). We also examined the mode of failure that occurred visually by using scanning electron microscopy. Seventy-two single rooted, recently extracted, premolar teeth were root canal treated and randomly divided into two groups. Group 1 was obturated with gutta percha and a calcium hydroxide-based sealer (Sealapex, Kerr). Group 2 was obturated with gutta percha and a eugenol-based sealer (Tubli-Seal Kerr). The teeth were stored for 1 week in distilled water at $37^{\circ} \mathrm{C}$ and then prepared for $9 \mathrm{~mm}$ posts with a $1 \cdot 40-\mathrm{mm}$ drill. The matching glass fibre post was luted with a resin cement following the manu- facturer's instructions. The samples were stored for 1 week and thermocycled. The posts were removed from the root canals using a calibrated testing machine in tensile mode. The mean dislodging force for group 1 was $190.46 \mathrm{~N}$ and for group 2 was $183.8 \mathrm{~N}$, with standard deviations of 54.9 and $56.0 \mathrm{~N}$ respectively. The $t$-test indicated no significant difference between the two groups. Failure of the posts occurred mainly within the resin layer. This study showed that under experimental conditions there was no statistically significant difference between Sealapex sealer and Tubli-Seal sealer on the retention of glass fibre posts using a resin cement. KEYWORDS: endodontics, resin cement, eugenol, glass fibre, endodontic post-dentine bonding

Accepted for publication 18 November 2005

\section{Introduction}

Endodontically treated teeth, which have discoloured or fractured crowns, may require full-coverage restorations. Often there is insufficient remaining tooth structure to retain a restoration and the endodontically treated tooth will require a post and core. A post's function is to retain the core, which replaces missing coronal tooth structure $(1,2)$. The clinical success of cast metal posts and cores is well documented (3), but occasionally they are aesthetically deficient.

Metal posts used to restore endodontically treated teeth may be visible through all-ceramic crowns and thin gingival tissues. Where there is a high lip line, the discolouration can result in a difficult aesthetic restorative problem. A non-metallic, tooth-coloured post, in combination with an all-ceramic crown, has aesthetic advantages over metal posts. The major advantage of a non-metallic post and core lies in its dentine-like shade, which will then contribute to the overall shade of the final restoration.

Furthermore, posts that have biomechanical properties similar to those of tooth structure may be advantageous (4, 5). Assif and Gorfil (6) reported that the interface of materials of differing moduli of elasticity can result in areas of increased stress concentration in root treated teeth. The literature suggests that the ability of post and core systems to protect the root from biomechanical failures may vary greatly (6-8). Fredriksson et al. (7) discussed the desirability of restoring endodontically treated teeth with non-metallic materials having physical properties and moduli of elasticity similar to that of dentine. The ability to use bonding agents for luting these restorations may further 
contribute to uniting the remaining tooth structure (9). Carbon fibre and glass fibre posts fracture 'favourably' (8), meaning that they fail under load before the tooth fractures (10). This makes re-restoration of teeth possible and manufacturers of these glass fibre posts claim that removing these posts is simple.

Eugenol has been reported to inhibit the free radical polymerization reaction of chemically cured composite resin (11-13). Inhibition has been attributed to the reaction of eugenol with free radicals associated with resin polymerisation. Eugenol may reduce the bond strength of resin to dentine and thus affect the retention of a post in a canal. However, there is conflicting evidence in this respect $(12,14)$. There are many dental products which contain eugenol, including endodontic sealers (for example, Tubli-Seal, Kerr, Grossman canal sealer, Sultan Chemists Inc.; PCA, Pulp Dent Corp). Many previous studies of post-retention omitted the relevant step of obturating the canal before post-preparation and luting. Thermocycling of samples was also not performed in many of these studies. Paschal et al. (15) demonstrated a significant decrease in post-retention in obturated versus unobturated teeth, showing the importance of obturating the canals when testing in vitro cements for post-retention. Furthermore, there is little evidence in the literature regarding glass fibre posts and few investigations regarding the effects of sealers or their constituents on non-metallic post-retention.

The purpose of this investigation was to examine the effects of eugenol- and non-eugenol-containing sealers on the retention of ParaPost Fibre White posts luted with ParaPost Cement. Secondly, to examine visually, and using scanning electron microscopy, whether cohesive or adhesive failure of the resin to dentine bond occurred.

\section{Materials and methods}

A pilot study was performed $(n=12)$ to test the methodology and to provide an estimate of sample size of the future study. A power calculation was also performed and determined that 72 specimens were needed to provide sufficient statistical data.

Recently extracted unrestored premolar teeth, which had been removed for orthodontic purposes, were stored in distilled water in a refrigerator except during manipulation. A single operator (SD) performed the root canal treatments using hand files. The crowns of the teeth were first sectioned $1 \mathrm{~mm}$ above the cementoenamel junction with a fine diamond disc* in a horizontal plane, perpendicular to the long-axis of the tooth. The teeth were prepared using a modified stepback technique. The canals were instrumented to a master file size 40 file $^{\dagger}$ and stepped back in $1 \mathrm{~mm}$ increments to file size 70 . Sodium hypochlorite $(5 \cdot 25 \%)$ was used for irrigation to reflect the clinical situation. Following irrigation the canals were dried using paper points.

The instrumented specimens were then randomly divided into two groups of 36 specimens. Group 1 had the root canals obturated with gutta percha ${ }^{\ddagger}$ and a noneugenol-containing sealer (Kerr Sealapex ${ }^{\ddagger}$ ) and group 2 had the root canals obturated with gutta percha and a eugenol-containing sealer (Kerr Tubli-Seal ${ }^{\ddagger}$ ). A lateral condensation technique with accessory gutta percha points was used in both groups.

After obturation, $3 \mathrm{~mm}$ of the gutta percha was removed from the root canal orifice and Cavit ${ }^{\S}$ temporary filling material was placed to seal the coronal orifice. The specimens were then stored for one week, submerged in distilled water at $37{ }^{\circ} \mathrm{C}$, which was to allow for complete set of the sealer before postpreparation. The teeth were stored in distilled water, based on the reports of two review articles $(16,17)$, which stated that for dentine adhesive research, stored teeth must be kept in a moist environment to prevent dehydration and should not be stored $>6$ months.

A $9 \mathrm{~mm}$ post-space was created in each specimen for a Size $5.5\left(0.055^{\prime \prime}\right.$ or $\left.1.40 \mathrm{~mm}\right)$ parallel sided, prefabricated ParaPost Fibre White post using the corresponding twist drill from the ParaPost XT kit ${ }^{\mathbb{T l}}$. The postspace was washed with distilled water for $10 \mathrm{~s}$ and dried with paper points. The parallel-sided posts were trial fitted in channels to confirm accurate depth and passive fit. The conditioner was mixed and applied for $30 \mathrm{~s}$ and dried, first with a coarse paper point to avoid pooling, and then lightly air dried for $2 \mathrm{~s}$. The conditioner was also painted onto the post using a brush.

The ParaPost Cement ${ }^{\text {II }}$ was mixed and applied according to the manufacturer's instructions. The resin cement was introduced to the canal using a coarse

\footnotetext{
*Superflex 355 514·220 Edenta; Au/SG, Switzerland.

${ }^{\dagger} \mathrm{K}$ Flexofile; Dentsply, Maillefer, Switzerland.

${ }^{\ddagger}$ Kerr Corp., Glendora, CA, USA.

$\S_{3 M}$ ESPE Dental Products, St Paul, MN, USA.

${ }^{\text {II }}$ ParaPost XT, Coltene, Whaledent, West Sussex, UK.
} 
paper point and a lentulo spiral filler was used to facilitate even filling of the dowel space. The corresponding ParaPost Fibre White post ${ }^{\text {II }}$ was coated with cement and placed into the channel to the pre-measured line and held with finger pressure until initial set. The excess cement was removed from the top of the tooth, so the cemented ParaPosts extended approximately $6 \mathrm{~mm}$ above the flat surface of the tooth. Both Group A and B posts were luted in this fashion by a single operator (SD). The cement was judged to have set using a probe on the specimen and by comparison of the material remaining on the mixing pad. The ParaPost Cement, when not in use, was refrigerated at $4{ }^{\circ} \mathrm{C}$, as recommended by the manufacturer.

Specimens were stored in distilled water at $37^{\circ} \mathrm{C}$ for a period of 1 week and then thermocycled 500× from $5{ }^{\circ} \mathrm{C}$ to $55^{\circ} \mathrm{C}$ with a dwell time of $10 \mathrm{~s}$ in each bath. The transfer time between cycles did not exceed 5-10 s. The roots were then notched and mounted in acrylic resin** in standardized acrylic moulds. A dental surveyor was used with a standardized mounting jig to ensure all posts were parallel to the future tensile force which would be used. This ensured that post-removal would occur in a direction parallel to the long-axis of the post.

A customized metal framework was made to support acrylic resin around the two rounded sections on the coronal part of the ParaPost Fibre White. This apparatus facilitated removal of the posts in tensile mode. Acrylic resin was used to attach this metal framework to the test specimen and the posts were then subjected to tensile force in the Hounsfield testing machine at $0.50 \mathrm{~mm} \mathrm{~min}^{-1}$. Data were recorded in Newtons and subsequently analysed using a paired $t$-test. Modes of failure were also noted. Sectioned root samples were further examined using scanning electron microscopy ${ }^{{ }^{+}}$ at 50 and $500 \times$ magnification.

\section{Results}

No root or post-fractures occurred in any of the samples during testing. Figure 1 displays the normal probability plots for groups 1 and 2, indicating that the data were normally distributed. Table 1 displays means and standard deviations for dislodging force for the two groups tested. The dislodging force for samples in group

\footnotetext{
**VariSet Met Prep; Coventry, UK.

${ }^{++}$Hitachi Scientific VP-SEM s-3500 N; Hitachi Corp, Ibarki, Japan.
}
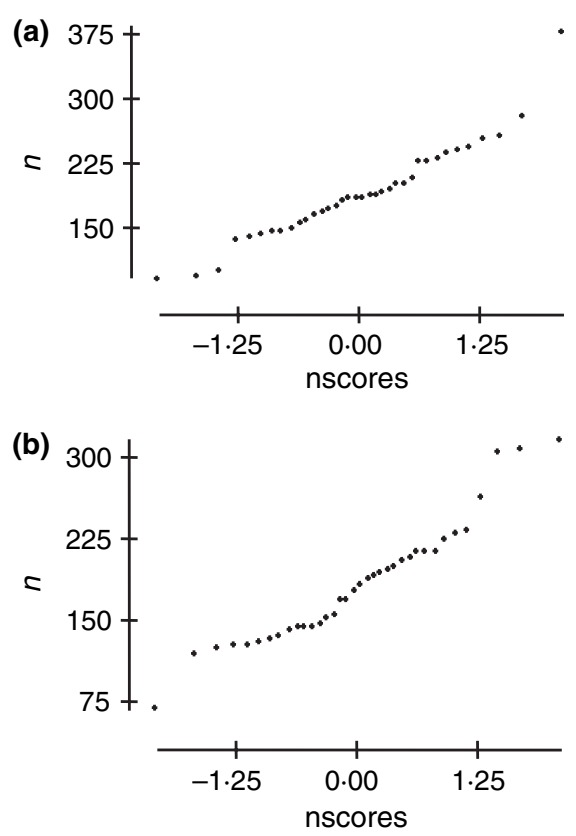

Fig. 1. Normal probability plots. The data from the non-eugenolcontaining group (a) and eugenol-containing group (b) are shown. A straight line indicates normal distribution of data.

Table 1. The means and standard deviations for dislodging force (N) for the two groups tested

\begin{tabular}{lcc}
\hline & Mean & s.d. \\
\hline Group 1 (non-eugenol-containing sealer) & $190 \cdot 46$ & $54 \cdot 85$ \\
Group 2 (eugenol-containing sealer) & $183 \cdot 84$ & $56 \cdot 04$ \\
\hline
\end{tabular}

1 was $190.5 \pm 54.9 \mathrm{~N}$ and for group 2 was $183 \cdot 8 \pm 56 \cdot 0 \mathrm{~N}$. A non-paired two-tailed student's $t$-test revealed that the two groups were not significantly different $(\mathrm{P}=0 \cdot 62)$.

Visual inspection of the specimens demonstrated fraying of the fibres towards the coronal end of the post with failure appearing to occur within the rootresin cement interface. Examination of sectioned root samples by scanning electron microscopy showed the root surface covered in a smear of debris (Figs 2-4). The mode of failure was mainly cohesive, with cement residue apparent on the root canal surface. In some areas, there were visible deposits of debris, thought to be primarily resin, and adjacent to these were smoother areas of root surface where the resin had come away more cleanly. This was attributed to failure occurring between the cement and the adhesive layer, with some cohesive failure occurring within the resin layer, to a 


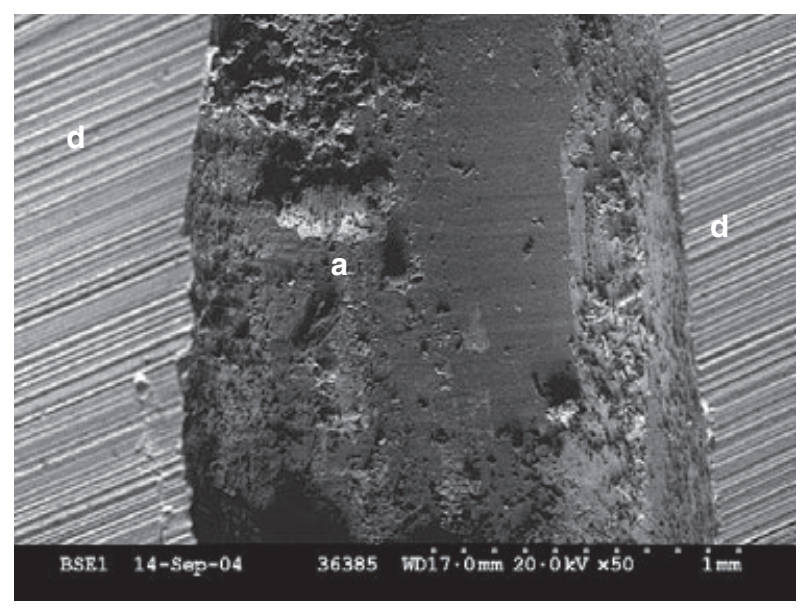

Fig. 2. Scanning electron micrograph $(\times 50)$ showing sectioned root following removal of post. The sectioned dentine surface (d) is visible and the remains of adhesive material (a) in the root canal.

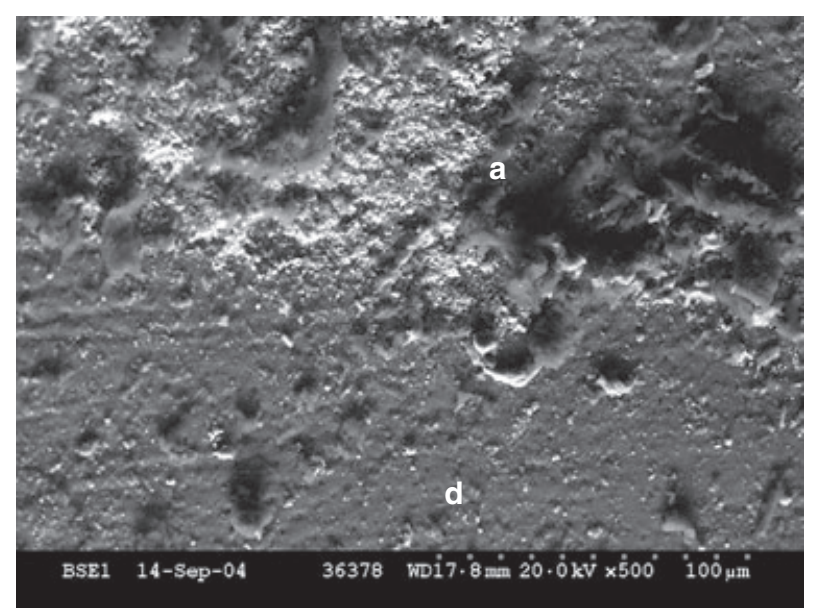

Fig. 3. Scanning electron micrograph $(\times 500)$ displaying failure of the adhesive material, with areas of resin and debris (a) adjacent to smooth areas of root surface (d).

lesser extent. This was the pattern for the majority of the samples viewed.

\section{Discussion}

Previous data indicate that residual eugenol could inhibit the setting of resin cement, weakening the bond, which retains glass fibre posts. Hence, the original expectation was that the non-eugenol-containing sealer group would have higher mean pull-out strengths than eugenol-containing group. No difference was found between the two groups in this study. No root fractures occurred in any of the specimens tested.

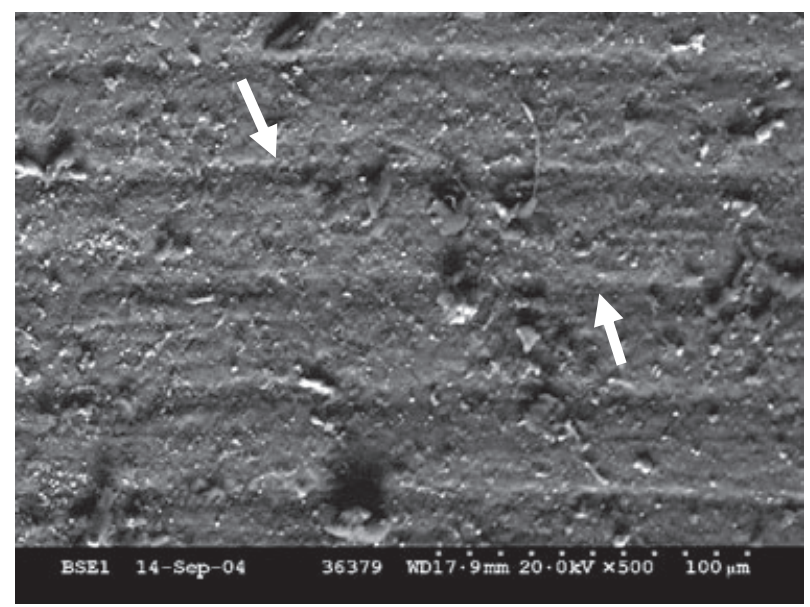

Fig. 4. Scanning electron micrograph $(\times 500)$ showing ridging (arrows) apparent on the smooth areas of root dentine.

Analysis visually, and using scanning electron microscopy, indicated that failure within the cement layer was the predominant mode of failure in both groups tested. This would suggest that the bond of the cement to the root canal dentine generally remained intact and thus, would appear not to be the limiting factor in retention. A similar finding was reported by Hagge et al. (5). Because no samples failed clearly at the root dentine-resin cement interface this would imply that there was effective removal of gutta percha and sealer components leading to effective dentine bonding. There were no post or root fractures noticed throughout the testing procedures in this study and the glass fibre posts were found to be extremely easy to adjust and remove from the canal, thus substantiating the manufacturer's claim of retrievability.

Following sectioning of the specimens, visual and scanning electron microscopy inspection revealed that resin cement appeared to wet the root canal uniformly with evidence of uniform cement distribution on the walls of the root canals. Minimal air trapping was noted and thus good wetting properties of the resin cement were demonstrated. The lack of dentinal tubules on examination with microscopy indicates good penetration of the resin cement into the dentinal tubules. Also evident in this study was an absence of voids or bubbles at the fibre post-resin interface, suggesting a good bond between the resin matrix of the post and of the resin cement. Apparent on several of the images were regular machined ridging which was thought to be due to post-preparation with the twist drill (Fig 4). 
Previous studies of post-retention have been performed, although many of these omitted the clinically relevant step of preparing post-spaces in previously root-filled canals. Paschal et al. (15) compared postretention of two cements in obturated (gutta percha with a eugenol based sealer) and unobturated canals and found significantly lower post-retention values in the canals that had been obturated, regardless of cement type used. Also, thermocycling was notably absent from many of the post-studies examined.

While the presence of a eugenol-containing sealer did not significantly reduce the bond strength in this study, this may not be true of another cement system. The results of this study concur with those of Schwartz et al. (12) and Boone et al. (18) who observed no difference between eugenol and non-eugenol-based sealers and resin cements. Other studies reporting comparable findings include Russo et al. (19) and Burns et al. (20), whereas Bergeron et al. (21) found a resin-based sealer resulted in higher post-retention values than a eugenolbased sealer. Here, conventional clinical techniques for luting a post in an endodontically treated tooth were adhered to as closely as possible. It is possible that during post-preparation, most or all of the eugenolbased sealer was removed. Some cleaning of the canal could also have occurred using distilled water. Thus, through mechanical and physical removal (flushing of the canal) the amount of debris and free eugenol available to affect the polymerization of the resin cement may have been reduced considerably. Boone et al. (18) stated that obtaining a clean bonding substrate may be the most critical factor in achieving success with resin cements and this was also substantiated in the present study. Also, it is expected that higher intra-oral temperatures may further increase the rate of polymer conversion and thus this luting agent demands expedient use. Working times were noticeably short throughout testing, although the resin cement was refrigerated when not in use.

Studies of post-retention $(14,22)$ do not always provide a clinically relevant model for examining the effects of eugenol on the retentive strength of resin cements. Paschal et al. (15) showed the importance of performing obturation procedures before canal preparation and thus it was appreciated that a study following conventional clinical techniques for root canal treatment was necessary.

It is acknowledged that in vitro bond strength testing may not be truly representative of the highly demanding intra-oral conditions and, at best, gives an indication of possible clinical performance of the materials tested. The validity of the results depends on how appropriate and representative the laboratory test conditions are. For that reason each step of the protocol was strictly adhered to and reflected the clinical procedure which would have been performed had this been an in vivo investigation. Although some retrospective studies exist, final conclusions regarding glass fibre systems will depend on the results of randomized-controlled clinical trials. Clinical long-term studies and investigations of failure of glass fibre posts in the intra-oral environment can best evaluate the lifespan and safety of these restorations.

In conclusion:

1 No statistically significant difference was observed in the retention strengths of ParaPost Fibre White posts luted with ParaPost resin cement in teeth where eugenol-containing and non-eugenol-containing root canal sealers were used.

2 The mode of post-failure appeared to be within the adhesive layer.

3 The retention strengths of glass fibre posts were comparable with those observed in studies of a similar nature.

4 The glass fibre posts used in this study proved easy to adjust and remove.

5 Limited working time was noted with the use of the resin cement used in this study.

6 Good wettability of the resin cement to the root was observed on SEM analysis.

\section{Acknowledgement}

The authors are grateful to Coltene/Whaledent, who generously provided the posts and resin cement used in this study.

\section{References}

1. Sorensen JA, Martinoff JT. Clinically significant factors in dowel design. J. Prosthet. Dent. 1984;52:28-35.

2. Caputo AA, Standlee JP. Biomechanics in Clinical Dentistry. Chicago, IL: Quintessence Publishing Company; 1987.

3. Morgano SM, Milot P. Clinical success of cast metal posts and cores. J. Prosthet. Dent. 1993;70:11-16.

4. Tjan AH, Grant BE, Dunn JR. Microleakage of composite resin cores treated with various dentine bonding systems. J. Prosthet. Dent. 1991;66:24-29.

5. Hagge MS, Wong RD, Lindemuth JS. Effect of three root canal sealers on the retentive strength of endodontic 
posts luted with a resin cement. Int. Endod. J. 2002;35: 372-378.

6. Assif D, Gorfil C. Biomechanical considerations in restoring endodontically treated teeth. J. Prosthet. Dent. 1994;71:565567.

7. Fredriksson M, Astback J, Pamenius M, Arvidson K. A retrospective study of 236 patients with teeth restored by carbon fiber-reinforced epoxy resin posts. J. Prosthet. Dent. 1998;80:151-157.

8. Akkayan B, Gülmez T. Resistance to fracture of endodontically treated teeth restored with different post systems. J. Prosthet. Dent. 2002;87:431-437.

9. Mendoza DB, Eakle WS, Kahl EA. Root reinforcement with a resin-bonded preformed post. J. Prosthet. Dent. 1997;78:10-15.

10. Mannocci F, Ferrari M, Watson T. Intermittent loading of teeth restored using quartz fiber, carbon quartz fiber, and zirconium dioxide ceramic root canal posts. J. Adhes. Dent. 1999;2:153-158.

11. Millstein PL, Nathanson D Effect of eugenol and eugenol cements on cured composite resin. J. Prosthet. Dent. 1983;50:211-215.

12. Schwartz R, Davis R, Hilton TJ. Effect of temporary cements on the bond strength of a resin cement. Am. J. Dent. 1992;5:147-150.

13. Paul SJ, Schärer P. Effect of provisional cements on the bond strength of various adhesive bonding systems on dentine. J. Oral. Rehabil. 1997;24:8-14.

14. Ganss C, Jung M. Effect of eugenol-containing temporary cements on bond strength of composite to dentin. Oper. Dent. 1998;23:55-62.
15. Paschal JEJ, Burgess JO, Robbins JW. Post retention with and without fillers. J. Dent. Res. 1997;76:412.

16. Soderholm KJ. Correlation of in vivo and in vitro performance of adhesive restorative materials: a report of the ASC MD156 Task Group on Test Methods for the Adhesion of Restorative Materials. Dent. Mater. 1991;7:74-83.

17. Rueggeberg FA. Substrate for adhesion testing to tooth structure - review of the literature. Dent. Mater. 1991;7: $2-10$.

18. Boone KJ, Murchison DF, Schindler WG, Walker WA 3rd. Post retention: the effect of sequence of post-space preparation, cementation time, and different sealers. J. Endod. 2001;27:768-771.

19. Russo EMA, Russo E, Carvalho RCR, Santos MG, Braga SRM. Effect of eugenol containing endodontic sealer on retention of posts. J. Dent. Res. 1999;78:389 (Abstract 2271).

20. Burns DR, Moon PC, Webster NP, Burns DA. Effect of endodontic sealers on dowels luted with resin cements. J. Prosthodont. 2000;9:137-141.

21. Bergeron BE, Murchison DF, Schindler WG, Walker WA., III Effect of ultrasonic vibration and various sealer and cement combinations on titanium post removal. J. Endodont. 2001;27:13-17.

22. Peutzfeldt A, Asmussen E. Influence of eugenol-containing temporary cement on efficacy of dentin-bonding systems. Eur. J. Oral. Sci. 1999;107:65-69.

Correspondence: Dr Brian C. O'Connell, School of Dental Science, Trinity College, Lincoln Place, Dublin 2, Ireland.

E-mail: boconne@dental.tcd.ie 